\section{P20 Characteristics and Eating Lifestyle of People Who Increased Family Meals During the State of Emergency Caused by COVID-19 in Japan}

Rie Akamatsu,DPH,RD, akamatsu.rie@ocha.ac.jp, Ochanomizu University, 2-1-1, Otsuka, Bunkyo-ku, Tokyo, Japan, 1120012; Misa Shimpo, PhD, RD, The University of Nagano; Yui Kojima, PhD, RD, University of Niigata Prefecture

Background: In April 2020, the Japanese government announced the first state of emergency for a month to prevent COVID-19. The measures taken during this period affect dietary patterns. Since people were requested to stay at home, the frequency of people eating with their family increased during this stage.

Objective: To examine the characteristics and changes in the eating lifestyle of people who increased family meals during the state of emergency.

Study Design, Settings, Participants: A cross-sectional study involving 6,000 individuals aged 20-65 years was conducted via an internet survey developed by a Japanese research company.

Measurable Outcome/Analysis: We compared the characteristics and changes of the eating lifestyle of participants before and during the state of emergency. Chisquared tests were used to compare 3 groups: people who increased, not changed, and decreased family meals during the state of emergency.

Results: A total of 1,293 (21.6\%) participants ate more with their families during the state of emergency. Compared to people who decreased or not the changed frequency of family meals, people who increased the frequency of family meals had a higher level of education, were female, were living with someone, and/or had a higher household income in 2019 (all $P<0.001$ ). They also ate a more nutritionally balanced diet, vegetables, and fruits, and felt that they changed to a healthier eating lifestyle during the state of emergency (all $P<0.001$ ). Furthermore, they were more likely to cook more and spend more at dinner and were less likely to eat out with their friends (all $P<0.001$ ).

Conclusion: Although the state of emergency was difficult for the entire population, people who ate more with their families during this period had healthier eating habits.

Funding: Health, Labour and Welfare Policy Research Grants, Special Research (grant number JP 20CA2040).

\section{P21 College Students Attending Online Programs Have Lower Rates of Food Insecurity than Residential Students}

OpeyemiE. Adewumi, BS, oeadewumi@crimson.ua.edu, University of Alabama, 403 Russell Hall, Box 870311, Tuscaloosa, AL, 35487; Linda L. Knol, PhD, RDN, University of Alabama; Rebecca L. Hagedorn, PhD, LD, RDN, Meredith College, Raleigh, NC 27607; Melissa D. Olfert, $D P H, R D N$, West Virginia University, Morgantown, WV

Background: Residential students living off-campus report greater food insecurity than those living on campus. Distance education programs are becoming popular and provide a way to continue one's education from home. The food security status of students completing their programs online is unknown.

Objective: To assess food security among college students by program type- residential or online.

Study Design, Settings, Participants: Cross-sectional study, students aged 18 years or older, attending a large Southern university completed an online survey in 2019.

Measurable Outcomes/Analysis: Food security status was assessed using the Adult Food Security Survey Module and students were classified as either food secure or insecure. Students belonged to either a residential or an online program. Binary logistic regression analysis was used to determine whether food security status differed by program type while adjusting for age, sex, race/ethnicity, family financial support, receipt of financial aid, and status as a first-generation student. Demographic and financial differences associated with food insecurity were compared by program type.

Results: Among students $(n=2,819), 43.1 \%$ were food insecure and $9.8 \%$ were online students. Food insecurity prevalence among the residential students was significantly higher than students obtaining their degree online $(44.3 \%$ vs $32.6 \%$, OR $=2.6,95 \% \mathrm{CI}=1.8,3.9)$. When compared to residential students, online students were more likely to be female $(\mathrm{OR}=3.6,95 \% \mathrm{CI}$ $=2.2,6.1)$, older $(\mathrm{OR}=1.2,95 \% \mathrm{CI}=1.1,1.2)$, married $(\mathrm{OR}=5.3,95 \% \mathrm{CI}=3.5,8.0)$, employed full-time $(\mathrm{OR}=$ $7.7,95 \% \mathrm{CI}=4.5,13.0)$ and less likely to receive financial aid $(\mathrm{OR}=0.5,95 \% \mathrm{CI}=0.4,0.8)$ and family support $(\mathrm{OR}=0.6,95 \% \mathrm{CI}=0.4,0.9)$.

Conclusion: The prevalence of food insecurity among students in this sample is twice as high as that of the state and nation. However, students in an online program may be more food secure compared to residential students. Further studies are needed to assess factors that may be associated with food insecurity among online students.

Funding: None.

\section{P22 COVID-19 Impacts on Self-Reported Food Safety Behaviors in Florida Youth}

Haaris Saqib, MA, hsaqib@ufl.edu, University of Florida, 2046 NE Waldo Rd, Ste 3156, Gainesville, FL, 32609; Crystal Almond, MS, MSE, University of Florida; Jose Garcia, $B S, B A$, University of Florida; Victoria Hunter Gibney, $M P H$, $C P H$, University of Florida; Karla Shelnutt, $P h D, R D$, University of Florida

Background: Measures to protect against the spread of COVID-19 include guidance on personal and environmental hygiene. Some guidelines related to COVID-19 directly align with or have similarity to evidence-based food safety practices (FSP) taught in nutrition education classes, including handwashing and washing produce before eating. 
P22 (continued)

Objective: To understand the impacts of the COVID-19 pandemic on youth handwashing and washing produce before eating behaviors.

Study Design, Setting, Participants: Data were collected from youth $(\mathrm{N}=1,098)$ in grades $3-5$ who participated in SNAP-Education (SNAP-Ed) and the Expanded Food and Nutrition Education Program (EFNEP) in Florida. Utilizing common self-report survey measures, baseline measures from 3 years prior to the pandemic and 3 months during the pandemic were compared for handwashing before eating and washing produce before eating. Due to incomplete data collection during the first 8 months of the pandemic, only behaviors from October 20-January 31 intervals (October 2018- January 2021) were compared to control for seasonal differences.

Measurable Outcomes/Analysis: Behavioral measures are based on a frequency scale from 1 to 4 (4 being the most frequent). An independent samples $t$ test was used to compare baseline FSP scores from intervals before and during the pandemic.

Results: No significant difference was found in the frequency of handwashing before and during COVID-19 (3.16 vs 3.18, respectively; $P=0.76$ ). A significant increase was found in the frequency of washing produce before eating before and during COVID-19 (3.14 vs 3.31, respectively; $P=0.008$ ).

Conclusion: This study did not find any significant changes in handwashing frequency during COVID-19, however, a significant increase in washing produce prior to eating was found. Handwashing prior to eating is a current CDC guideline for COVID-19 mitigation while washing produce prior to eating is not (CDC 2021). Changes in lifestyle and/or eating arrangements during the pandemic might explain these findings.

Funding: Supplemental Nutrition Assistance Program Education.

\section{P23 Dietary Changes Among Japanese Adults Since the Spread of COVID-19}

Misa Shimpo,PhD, RD, shimpo.misa@u-nagano.ac.jp, The University of Nagano, 8-49-7, Miwa, Nagano, Nagano, Japan, 380-8525; Rie Akamatsu, RD, DrPH, Ochanomizu University; Yui Kojima, $P h D, R D$, University of Niigata Prefecture

Background: Preventive measures adopted for COVID19 have brought about significant changes in lifestyle and have affected dietary habits worldwide.

Objective: To examine the factors relating to dietary changes among Japanese adults since the spread of COVID-19.

Study Design, Settings, Participants: A cross-sectional questionnaire survey was conducted via the internet in November 2020. All 6,000 participants, comprising 3,044 male and 2,956 female adults, aged 20-64 years, who registered with a research company received the questionnaire on email.

Measurable Outcome/Analysis: The questionnaire included items on demographic characteristics; socioeconomic factors; past medical history; COVID-19 infection situation of their family and neighbors; fear of COVID-19; and lifestyle habits and healthy changes in dietary habits since the spread of COVID-19. The participants were given multiple choices in answers: changed to healthier; changed to unhealthier; and no-change. Multinomial logistic regression analysis was conducted to examine factors associated with dietary changes.

Results: Of the participants, 1,215 (20.3\%) answered that their dietary habits became healthier, 491 (8.2\%) answered as unhealthy, while a majority of them $(4,294$; $71.6 \%)$, said their dietary habits remained unchanged. The results of the multinomial logistic regression analysis with unchanged as the reference indicated that healthier and unhealthier dietary change were negatively associated with age (OR [95\% CI]: 0.89[0.84-0.95], 0.89 [0.81-0.97]) and positively associated with the past medical history of dyslipidemia (1.44 [1.11-1.86], 1.74 [1.23-2.46]) and fear of COVID-19 (1.05 [1.04-1.07], 1.02 [1.01-1.04]). Unhealthy change was positively associated with living alone, COVID-19 infection of colleagues, stress, and BMI; while annual household income, COVID-19 infection of oneself and friends, health literacy, frequency of exercise, and smoking were positively associated with healthier dietary changes.

Conclusion: This study suggested that the factors determining healthy and unhealthy dietary change since the spread of COVID-19 were age, COVID-19 infection, and fear of COVID-19.

Funding: Health, Labour and Welfare Policy Research Grants, Special Research (Grant Number JP 20CA2040).

\section{P24 Effect of a Nudge Intervention for Increasing Vegetable Intake Among University Students in a University Cafeteria}

Makiko Nakade,PhD, RD, nakade@shse.u-hyogo.ac.jp, University of Hyogo; Research Institute for Food and Nutritional Sciences, 1-1-12 Shinzaike-honcho, Himejicity, Hyogo, Japan, 6700092; Mako Matsushita, University of Hyogo; Ryota Fukui, BS, University of Hyogo; Kokoro Nakamura, University of Hyogo; Miho Fujishiro, University of Hyogo

Background: An intake of $\geq 350$ g of vegetables per day is recommended in Japan. However, the average intake of vegetables among adults $\geq 20$ years is about $280 \mathrm{~g}$ in 2019 , and the intake of young adults in their 20 s, including university students, is low (about $223 \mathrm{~g}$ ). Recently, the use of nudge strategies for behavioral change has been gaining attention. However, relatively few nudge trials for increasing vegetable intake have been conducted in Japan. 